\title{
Stress fracture of the femoral neck in a marine trainee female: case report
}

\begin{abstract}
Stress fractures of the femoral neck are uncommon, accounting for $5 \%$ of all stress fractures, being one of the most severe and disabling complications in training military personnel. This could bring potential serious consequences if an early diagnosis and treatment is not accomplished.
\end{abstract}

We present the case of a neck stress fracture in a young 25 -year-old military female, treated by reduction and internal fixation. She was followed up for two years and is reported without a vascular necrosis of the femoral head, without osteoarthrosis data and adequate bone consolidation.

This article aims to provide the first contact physician with the necessary tools for the early recognition of this type of injury so that it could predict the progression and displacement of the fracture, as well as significantly reduce the risk of devastating complications such as a vascular necrosis.

Keywords: femoral fracture, marine trainee, stress fracture

\author{
Volume 8 Issue I - 2018 \\ Josafat Yair Arroyo-Aparicio,' Bertina Diaz de \\ Jesús, ${ }^{2}$ Maria Ignacia Galicia Cornejo, ${ }^{3}$ Afrania \\ Zamora Lizarraga, ${ }^{4}$ Victor García García, ${ }^{5}$ \\ Hugo Iván Ortega Barnet, ${ }^{5}$ José Ernesto Paz \\ Zamora, ${ }^{5}$ Gamaliel Jaime Hernández, ${ }^{6}$ Cesar \\ Manuel Vargas Sahagún, 7 Medina Andrade \\ Luis Angel \\ 'Orthopedic Surgery Resident, Postgraduate School in Naval \\ Health, Centro Medico Naval, Universidad Naval, Mexico \\ ${ }^{2}$ Orthopedic Surgery Chief, Professor of the residence of \\ Traumatology and orthopedics, High Specialty Naval Health \\ Hospital, Centro Medico Naval, Universidad Naval, Mexico \\ ${ }^{3}$ Orthopedic Surgeon with high specialty in acetabulum, hip and \\ pelvis, Postgraduate School in Naval Health, Centro Medico \\ Naval, Universidad Naval, Mexico \\ ${ }^{4}$ Anesthesiologist, Hospital General Regional \#2 of Traumatology \\ and Orthopedics, Mexico \\ ${ }^{5}$ Postgraduate School in Naval Health, Centro Medico Naval, \\ Universidad Naval, Mexico \\ ${ }^{6}$ Orthopedic Surgeon, Centro Medico Dalinde, Mexico \\ ${ }^{7}$ General Surgery Department, Hospital General Regional IA, \\ Rodolofo de Mucha Macias, IMSS, Mexico
}

\begin{abstract}
Correspondence: Luis Angel Medina Andrade, General Surgery Department, Hospital General Regional, México, Email buismedina_5@hotmail.com
\end{abstract}

Received: February 07, 2018 | Published: February 15, 2018

\section{Introduction}

A stress fracture is the one secondary to overuse, that results for repetitive damage with load cycles lower than the stress required fracturing the bone, resulting in micro fractures. They are related to intense exercise, especially that involving repetitive weight-bearing loads, like military training or running. They occur when bone is unable to repair the repetitive damage. ${ }^{1}$

This kind of fractures is especially frequent in military training compared with athletic programs and represents the most important cause of hospital admission during military training with the subsequent loss of life quality. ${ }^{1}$

The risk factors are multiple, but some of them had been clearly identified as intrinsic or extrinsic. The first include aerobic fitness, hormonal and nutrition factors and gender. The gender and physical conditioning prior to the time of beginning training have been identified as an important risk factor, and extrinsic factors include factors related to physical training, especially volume and intensity of training. ${ }^{1}$

\section{Case report}

Female patient of 25years-old, active military, size $165 \mathrm{~cm}$, weight $52 \mathrm{~kg}$, BMI 19.1, with antecedent of military training for about
5 to 6hours a day, 6days a week, for 2 months prior to the onset of symptomatology. She refers with discrete right inguinal pain, which increased with physical effort, radiating towards the proximal third of the thigh and right buttock. It is evaluated by a general physician and diagnosed as a muscle contraction, without abnormalities in the $\mathrm{x}$-ray exam (Figure 1) indicating treatment with analgesics and nonsteroidal anti-inflammatory drugs, without suspension of physical activities. She comes back to the emergency department of the Naval General Hospital of High Specialty one week later, referring an acute increase in the symptoms and limitation of movement due to pain, an antero posterior pelvic radiograph is performed, without data of apparent bone lesion (Figure 2).

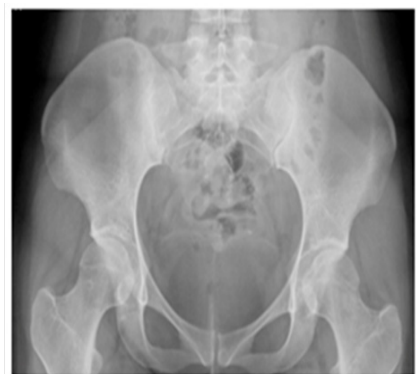

Figure I 24/08/20I5 anteroposterior pelvic radiograph, taken on the day of admission, with no data of apparent bone lesion. 


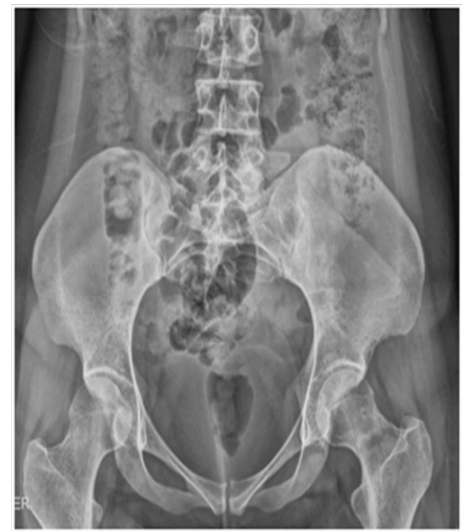

Figure 2 01/09/2015 anteroposterior pelvic radiograph, taking in the follow up, without no data of apparent bone lesion.

General surgery service is consulted by the inguinodynia diagnosis, but the need for surgical treatment is discarded, and patients are discharged with a diagnosis of muscle contracture of the hip abductors, going under treatment with physical therapy, rehabilitation, and analgesics.

After a month without improvement, the orthopedics service is consulted again. At physical exam, the patient presented shortening of about $2 \mathrm{~cm}$ of the right pelvic limb and limitation due to pain in the right hip mobility arches. A new antero posterior pelvic radiograph is requested, finding a right femoral trans-cervical continuity solution, without displacement, with varus angulation and cervical-diaphyseal angle of $117^{\circ}$ (Figure 3 ).

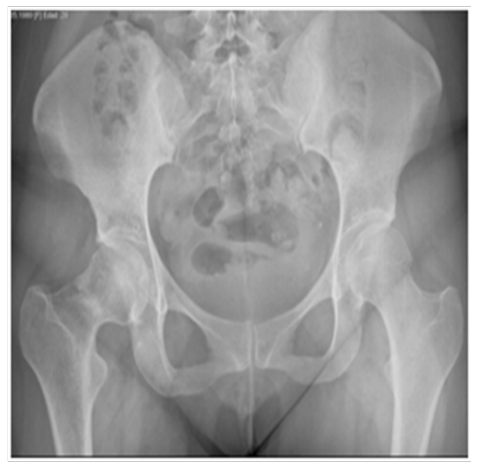

Figure 3 14/10/2015 pelvic anteroposterior radiography one month after the initial date of admission. A right femoral transcervical continuity solution was observed, with no varus angulation and a cervical-diaphyseal angle of $17^{\circ}$.

During the hospital stay, hormonal profile and bone densitometry were performed and bone metabolism alterations discarded. Magnetic resonance image of the lumbar spine was performed to rule out a neuropathic pathology but was negative too.

After study protocol patient was treated with surgical reduction and internal fixation with two cannulated screws, recovering diaphyseal cervical angle at $130^{\circ}$ (Figure 4), and starts with partial discharge after two weeks of the surgery. Bone callus formation is identified two months after surgery (Figure 5), initiating total support of the limb 3 months after surgery, and at 6months he fully reincorporates her activities with excellent clinical evolution. After two years of followup on his control radiographs, no avascular necrosis of the femoral head was observed and adequate bone remodeling appreciated (Figure $6)$.

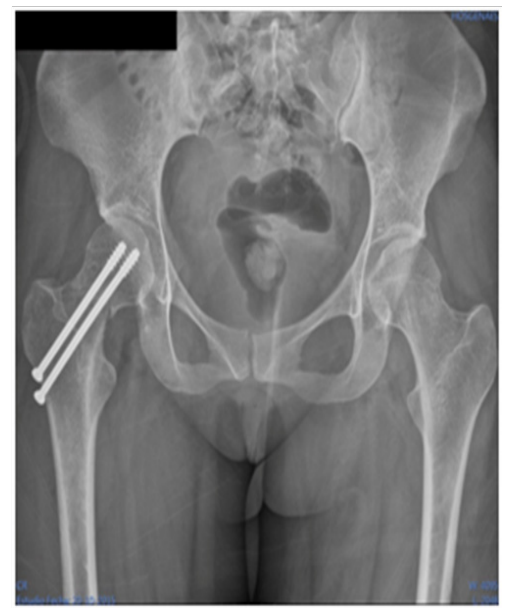

Figure 4 20/10/20I5 pelvic anteroposterior radiograph after surgical reduction and internal fixation with two cannulated screws.

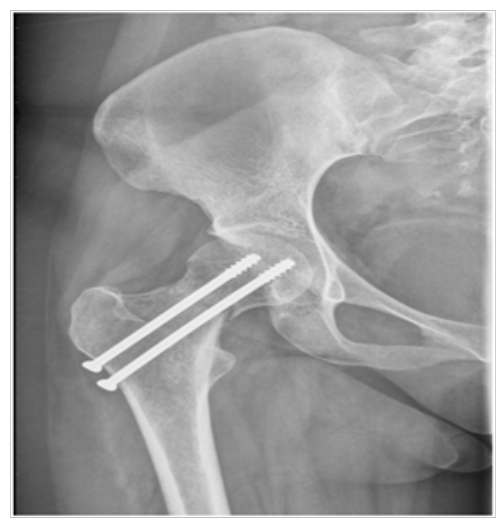

Figure 5 27/II/20I5 Radiograph two months after surgery with callus formation.

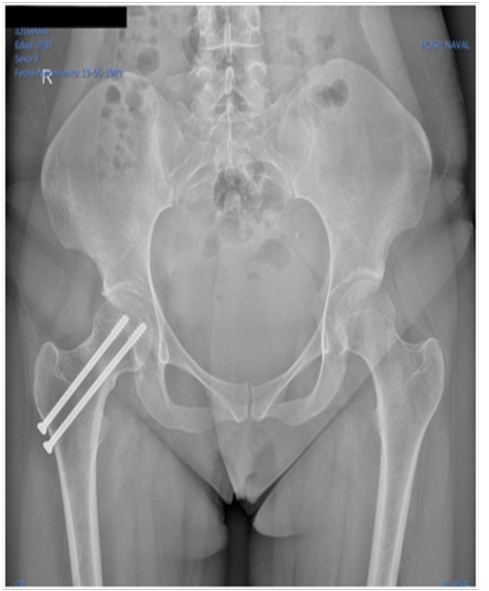

Figure 6 23/10/2017 pelvic anteroposterior radiograph two years after surgery with adequate bone remodeling.

\section{Discussion}

A stress fracture is the one that occurs secondary to the inability of the bone to support a repetitive applied force of a lower magnitude than the fracture threshold but generating gradual alteration of the bone structure. The osseous tissue has the capacity to remodel and adapt to changes in the magnitude and distribution of the forces acting 
on it. However, this process is insufficient compared to the repeated application of high loads, which are capable of generating trabecular microfractures. ${ }^{2}$

Fatigue fracture of the femoral neck mainly affects young patients, it appears in the military during periods of intensive instruction and in athletes who perform an increase in their frequent physical activity, although stress fractures are relatively common in this type of patient, it is estimated that only $1 \%$ of these fractures affect the femoral neck, there is usually no previous traumatic event and when there is no displacement of the fracture the symptoms can be very unclear and non-specific, and frequently the initial radiographs are normal, which leads to a delay in the diagnosis and treatment. ${ }^{2,3}$ The most important for the diagnosis of this injury is to suspect its existence, being the most common symptoms the inguinal pain that is exacerbated by physical activity and decreases with rest, with an special increase incidence in females. ${ }^{1,3}$

The delay in the diagnosis of this condition is common, several studies report an average of 14 weeks of delay for the diagnosis, two weeks more than in the present case. ${ }^{4,5}$ The displacement of stress fractures is the factor of greatest determination and prognosis for patient evolution, is responsible for $60 \%$ of the reduction of physical and sports activities in the patient, and carries a $30 \%$ incidence for a vascular necrosis, one of the most devastating results for this kind of population and their lifestyle, with the subsequent importance of medical staff knowledge about this pathology and initial presentation, for an early suspect and promptly treatment. ${ }^{6}$

\section{Conclusion}

Early recognition of stress fractures in high risk population could predict the progression and displacement of the fracture and significantly reduce the risk of devastating complications such as a vascular necrosis, especially in young patients with the previously mentioned lifestyle. This document highlights the significance of stress fractures of the femoral neck in the military in intense training, alerting first-contact physicians to suspect this type of injury in a high-risk population, which could prevent serious sequelae in these patients.

\section{Acknowledgements}

None.

\section{Conflict of interest}

The author declares no conflict of interest.

\section{References}

1. Kunte R, Basannar D, Chatterjee K, et al. Gender differential and implications in the epidemiology of stress fractures among cadets of Indian Armed Forces. Med j med forces India. 2017;73(4):356-362.

2. Kevin L, Michael B, Cory AC. Preoperative decision making in the treatment of high-angle "Vertical" femoral neck fractures in young adult patients an expert opinion survey of the orthopedic trauma association's (OTA) Membership. J Orthop Trauma. 2014;28(9):221-225.

3. Talbot JC, Cox 2 G, Townend M, et al. Femoral neck stress fractures in military personnel - a case series. Army Med Corps. 2015;154(1):47-50.

4. Konetsky M, Miller J, Tripp C. Femoral neck stress fracture. journal of orthopedic\& sports physical therapy. 2013;43(4):275.

5. Davey T, Lanham-New b SA, Shaw AM, et al. Fundamental differences in axial and appendicular bone density in stress fractured and uninjured Royal Marine recruits - A matched case-control study. Elsevier Inc Bone. 2015;73:120-126.

6. Cosman F, Ruffing J, Zion M, et al. Determinants of stress fracture risk in United States Military Academy cadets. Elsevier Inc Bone. $2013 ; 55: 359-366$. 